\title{
MOTIVOS Y EXPECTATIVAS DE LA DEMANDA DE APOYO PEDAGÓGICO EN EDUCACIÓN SECUNDARIA
}

\author{
Motives and expectations of the demand for pedagogical support
}

\section{in secondary education}

\author{
https://doi.org/10.22235/pe.v10i2.1423
}

\section{AGUSTINA MARÍA MANAVELLA*1 ROCÍO BELÉN MARTÍN *2}

Consejo Nacional de Investigaciones Científicas y Técnicas,

Recibido: 19/12/16

Universidad Nacional de Villa María. Argentina.

Revisado: 21/03/17

Correspondencia: agumanavella@gmail.com, rociobelenmartin@gmail.com

Aceptado: 06/04/17

Resumen: Con el propósito de conocer las demandas externas a la institución educativa, este artículo aborda el apoyo pedagógico como un contexto educativo que emerge del entrecruzamiento entre los contextos formales y no formales de aprendizaje, y que produce una relación de refuerzo y colaboración. Este estudio pretende reconocer los motivos por los que las familias solicitan el apoyo pedagógico en el primer año de la educación secundaria. Para ello, se realizaron 15 entrevistas en profundidad a las madres de estudiantes que asistieron a clases de apoyo, a las maestras que brindaron el servicio y a diferentes agentes educativos de un colegio ubicado en un pueblo del sur de Córdoba, Argentina. Se encontró que los motivos por los que los familiares demandan los contextos de apoyo pedagógico están atravesados por cuestiones de índole académica, socio-familiar y afectiva. Esto permite pensar las clases de apoyo como un contexto de educación que fortalece oportunidades de aprendizaje.

Palabras clave: enfoque socio-cultural, aprendizaje, contextos de aprendizaje, apoyo pedagógico, educación secundaria.

\begin{abstract}
With the purpose of knowing the demands that happen outside of educational institutions, this article approaches the pedagogical support as an educational context that emerges from the intersection between the formal and non-formal contexts of learning, and that produces a relationship of reinforcement and collaboration. This study aims to recognize the reasons why families ask for pedagogical support in the first year of secondary education. To this end, fifteen in-depth interviews were conducted with the mothers of students who attended support classes, the teachers who provided the service and different educational agents of a school located in a town in the south of Cordoba, Argentina. It was found that the reasons for which the relatives demand the contexts of pedagogical support are crossed by issues of academic, socio-familiar and affective nature. This study allows to think of support classes as an educational context that strengthens learning opportunities.
\end{abstract}

Keywords: socio-cultural approach, learning, learning contexts, educational support, secondary education.

\footnotetext{
${ }^{1}$ Licenciada en Psicopedagogía (Universidad Nacional de Villa María, Argentina) y psicopedagoga (Escuela Normal Víctor Mercante, Argentina). Becaria de investigación doctoral del CONICET (Universidad Nacional de Villa María, Argentina). Su investigación está centrada en el aprendizaje en contextos no formales, de capacitación para el trabajo.

2 Doctora en Psicología (Universidad Nacional de San Luis, Argentina) y licenciada en Psicopedagogía (Universidad Nacional de Río Cuarto, Argentina). Becaria de investigación posdoctoral del CONICET (Universidad Nacional de Villa María, Argentina). Su investigación está centrada en las comunidades de aprendizaje, comunidades de práctica, aprendizaje situado y contextos de aprendizaje formales, no formales e informales.
} 


\section{INTRODUCCIÓN}

Los espacios no formales de aprendizaje, como extensión y alternativa a las formas tradicionales de enseñanza, fueron reconfigurado las interpretaciones sobre el aprendizaje de las personas (Martín, 2014). Las clases de apoyo pedagógico, un tipo de contexto no formal, en ocasiones son denominadas la sombra del sistema educativo, ya que acompaña a la educación formal como si fuese una penumbra y se configura como respuesta y solución a aquellas cuestiones que la educación formal no puede atender debido a la multiplicidad de funciones educativas que demanda la sociedad.

El estudio que aquí se presenta se llevó a cabo siguiendo los lineamientos de los estudios de caso, mediante la administración de entrevistas a familiares de estudiantes de primer año de educación secundaria, a maestras de apoyo pedagógico y a diversos agentes educativos de una institución de la provincia de Córdoba, Argentina.

\section{Algunas consideraciones sobre el apoyo pedagógico}

Dentro del campo de la psicología educacional, los estudios sobre contextos no formales de aprendizaje y las contribuciones referidas al apoyo pedagógico resultan relevantes para comprender el fenómeno de la demanda familiar de dichos contextos. Desde esta disciplina, Rinaudo (2014) realiza un recorrido sobre la temática y describe las concepciones sobre los contextos de aprendizaje en el marco de los enfoques conductistas, constructivistas cognitivos y socioculturales. En su trabajo, resalta la importancia de los vínculos entre las personas, las demandas de la tarea, los propósitos de la acción y las herramientas, materiales o simbólicas, disponibles para la generación de contextos de aprendizaje promisorios.

Los contextos no formales de aprendizaje representan aquellas actividades educativas organizadas, sistemáticas, realizadas fuera del marco del sistema oficial. Se refiere entonces a las instituciones, actividades, medios y ámbitos de educación que, sin ser escolares, han sido creados para satisfacer determinados objetivos educativos. Estos ambientes se consideran importantes para facilitar los aprendizajes en grupos particulares de la población. Asimismo, los contextos no formales se distinguen por su carácter final: no dan salida a niveles o grados educativos, sino más bien al entorno social y productivo, por su potencial flexibilidad y funcionalidad respecto de los programas y métodos (Martín, 2014; Smitter, 2006; Trilla, Gros, López y Martín, 2003).

Desde estos planteos, el apoyo pedagógico es considerado un tipo de contexto no formal. Autores como Stevenson y Baker (citado en Bray, 1999) lo definen como un servicio educativo de carácter privado, que se brinda fuera del sistema educativo formal $y$ en el que se desarrollan actividades similares a las escolares para mejorar los resultados académicos del alumnado que transita la enseñanza primaria o secundaria. 
Bray (citado en Runte-Geidel, 2013) define dos criterios para caracterizar los tipos de apoyo pedagógico escolar: el carácter complementario, que está centrado en clases que tratan contenidos y materias desarrollados en la escuela, y el carácter privado en el que, como su denominación lo indica, las clases son suministradas a cambio de un pago.

Diversas investigaciones (Bray, 1999; Bray, Adamson y Mason, 2010; Buchmann, 2002; Runte-Geidel, 2015) han reflejado los distintos motivos por los cuales las personas acuden al contexto de apoyo pedagógico. Al respecto, Ventura, Neto-Mendes y Costa (2006) consideran las siguientes funciones de este contexto educativo:

- El apoyo al aprendizaje fuera de la escuela puede permitir (aunque no siempre ocurra así) una enseñanza individualizada, lo que contrasta con la enseñanza masificada ofrecida por los sistemas educativos modernos.

- Pueden presentarse como el espacio de realización de los trabajos de casa, lo que muestra una dimensión de la complementariedad que puede existir con el trabajo que se realiza en el sistema regular de enseñanza.

- Pueden aun realizar la función (quizás una de las más apreciadas por los clientes en estos servicios) de preparación para los exámenes, cuya relevancia máxima se alcanza en el examen nacional.

- Las explicaciones pueden cumplir la función (más social que académica) de apoyo a la familia, ofreciendo servicios de ocupación de los tiempos libres vitales para una familia nuclear cada vez más restringida y con elevados índices de ocupación laboral fuera de la esfera doméstica (Ventura et al., 2006, p. 3).

Agalianos (2011), en tanto, expresa que algunos familiares demandan clases particulares para sus hijos porque consideran que estos pueden sentirse más a gusto en grupos más reducidos de trabajo. Estos grupos crean un ambiente que les permite a los estudiantes hacer preguntas sin exponerse a situaciones de acoso escolar por parte de sus compañeros cuando muestran desconocer algunos temas o presentan un ritmo de aprendizaje más lento.

En algunos países de Europa, específicamente en Grecia, la asistencia de los estudiantes a clases particulares ha estado asociada a la ocupación de ambos padres y a su escasa disponibilidad horaria para ayudar a los hijos con las tareas escolares. En Luxemburgo, los centros de tutorías privadas son atractivos para los padres que no tienen tiempo durante el día para cuidar a sus hijos. El hecho de que asistan a clases particulares deja a los familiares tranquilos, ya que asumen que allí harán los deberes y estarán bien cuidados (Agalianos, 2011). 
En el mismo sentido, Ficetti (citado en Moraga, 2011) plantea que muchos padres solicitan las clases de apoyo pedagógico por necesidad laboral, pero otros tercerizan la función materna y paterna. En su escrito, Ficetti también hace mención a la devaluación que ha sufrido en los últimos tiempos la figura del docente.

Runte-Geidel, en una investigación que realizó en el año 2015, señala que la mayoría del alumnado de secundaria busca el recurso de las clases particulares como refuerzo para los contenidos impartidos en las escuelas y para su mejor desempeño académico. Esto demostraría que la educación formal no resulta suficiente para que los estudiantes alcancen los resultados esperados. Agalianos (2011) considera que las clases de apoyo pedagógico son menos desarrolladas por alumnos que están en verdadera necesidad de ayuda escolar, y más solicitadas por familiares de estudiantes que buscan mantener sus calificaciones.

Este artículo se enfoca en las demandas que los familiares realizaron de los contextos de apoyo pedagógico ofertados fuera de la institución educativa, en los que dos maestras de clases particulares brindaron apoyo y trabajaron con los contenidos curriculares propios del primer año de educación secundaria. Debido a que este contexto era demandado sobre todo para repasar los contenidos curriculares la semana anterior a las evaluaciones, las actividades que se llevaron a cabo en las clases consistieron en ejercicios en los que las maestras les brindaban a los jóvenes situaciones problemáticas similares a las que habían trabajado en clases, para que las practicaran y resolvieran. En el caso de los estudiantes que asistieron a clases particulares a prepararse para materias con contenidos curriculares teóricos, las actividades correspondieron a la implementación de diversas técnicas de estudio —-tales como resúmenes y cuadrospara que pudieran repasar el contenido en sus hogares.

\section{ASPECTOS METODOLÓGICOS}

Con el propósito de comprender en profundidad los motivos por los cuales los familiares solicitan apoyo pedagógico en el momento en que sus hijos transitan la educación secundaria, se desarrolló este estudio de acuerdo a la metodología de los estudios de caso (Stake, 1998).

\section{Delimitación del caso}

La institución educativa en la que se realizó el estudio está ubicada en un pueblo del sur de Córdoba (Argentina), es de gestión privada y brinda educación a 120 adolescentes. Como el propósito era investigar un curso de primer año, se tomó como muestra uno que se desarrolló durante 2015, a fin de poder contar con información acerca de la demanda de apoyo a lo largo de un año y en sus diferentes momentos.

El curso seleccionado estuvo conformado por 41 alumnos que provenían de cursar sus estudios desdoblados en dos salas: A y B. Durante el cursado de primer año, 
los jóvenes tenían diez materias y asistían al colegio cinco horas diarias, de lunes a viernes de 08:25 a 13:25. Por la tarde, los martes y jueves tenían una hora de Educación Física, y los miércoles tenían dos horas destinadas a un taller extracurricular de Estrategias de Aprendizaje.

\section{Participantes}

El caso de estudio estuvo conformado por 15 madres de estudiantes -que demandaron apoyo pedagógico durante el cursado de primer año en 2015- dos maestras de apoyo y otros agentes educativos (directora y psicopedagoga).

\section{Materiales y modalidad de recolección de datos}

Los datos fueron recolectados mediante entrevistas individuales y semiestructuradas, lo que permitió que las personas pudieran hablar de sus experiencias, sensaciones e ideas (Stake, 1998). Previamente, con autorización de las autoridades del centro educativo para poder contactar a los entrevistados, se realizó una encuesta que informó a los familiares sobre la temática investigada. En ella se solicitó a un adulto que respondiera si durante el cursado de primer año en 2015 había demandado la asistencia del adolescente a clases particulares.

\section{Procedimientos}

El trabajo de campo se inició a través de la entrevista con la directora del establecimiento. En esa oportunidad se informó a la autoridad sobre la temática a investigar y se solicitó su permiso para acercarle a cada alumno una encuesta. Luego se concretó la entrevista con la psicopedagoga del colegio. En paralelo, se realizaron entrevistas con las maestras de apoyo pedagógico y se envió la encuesta a los hogares de los estudiantes que cursaron primer año durante 2015. A partir de 24 encuestas contestadas, se procedió a realizar las entrevistas con los familiares que habían demandado el contexto de apoyo pedagógico.

\section{RESULTADOS Y ANÁLISIS}

El presente estudio se desprende de una investigación de mayor amplitud ${ }^{3}$ en la que se estudió la demanda familiar de contextos de aprendizaje extraescolares en educación secundaria. Aquí se hará hincapié en el análisis de una de las categorías de esa investigación, que refiere a los motivos familiares para la demanda de apoyo pedagógico.

\footnotetext{
${ }^{3}$ Se trata de un trabajo final de grado de la Licenciatura en Psicopedagogía, realizado en la Universidad Nacional de Villa María, Córdoba, Argentina (Manavella, 2016).
} 
De 17 encuestas en las que los familiares respondieron que los adolescentes habían asistido a clases particulares, y que estaban dispuestos a ser entrevistados, se realizaron 15 entrevistas. En dos casos no se pudo concretar el encuentro con los familiares de los jóvenes. De los familiares entrevistados, cuatro respondieron que sus hijos habían asistido a clases de apoyo durante el cursado de los estudios primarios, mientras que el resto comenzó a demandar este contexto al ingresar a la educación secundaria.

Respecto a los motivos por los que se demandaron los contextos de apoyo pedagógico, se han encontrado variadas respuestas. Aquí se incluyen no solo las respuestas de los familiares, sino también fragmentos de las entrevistas a las maestras de apoyo pedagógico, la directora y la psicopedagoga. Las respuestas a las preguntas fueron agrupadas según el motivo por el que se demandaba el contexto de aprendizaje. Los motivos son diversos, pero se aglutinan por aspectos similares que hacen a cuestiones de índole académica, afectiva y socio-familiar.

De acuerdo a los resultados, el contexto de apoyo pedagógico fue demandado principalmente por los siguientes motivos: (a) necesidad de ayuda en el proceso de adaptación, (b) falta de tiempo por parte de los familiares y (c) conocimientos insuficientes de los familiares. A continuación, se describen y explican en profundidad cada uno de ellos.

\section{Ayuda al proceso de adaptación}

Diez familiares respondieron que el motivo por el que demandaron clases particulares fue para que los adolescentes pudieran adaptarse al pasaje de un nivel educativo a otro. El pasaje a primer año, sumado a los cambios que acontecen en relación a la educación primaria - tales como la presencia de un número mayor de asignaturas, la cantidad de profesores, el incremento de la carga horaria - parecen generar que los estudiantes se encuentren desorientados en relación a la nueva modalidad de enseñanza. Esto trae aparejado que sus familiares requieran de un maestro de apoyo pedagógico para que ayude a los jóvenes a organizarse y a responder a las exigencias de la educación secundaria. Se trata entonces de un motivo de índole académica. La ruptura que implica el pasaje de los estudios primarios a los secundarios se ve reflejada en el aumento de la asistencia a clases de apoyo pedagógico en el momento de transición entre un nivel de enseñanza y otro.

Algunos familiares enfatizaron que demandaron este apoyo por la necesidad de adaptación a las dinámicas que se manejan en los diferentes niveles educativos. En otros casos, los familiares relacionaron las dificultades en la adaptación del pasaje de un nivel educativo a otro con la calidad de formación del nivel primario. Los miembros de la institución educativa respondieron en la misma dirección que los familiares, haciendo hincapié en los cambios que implica el pasaje de nivel en relación a la carga horaria, la 
cantidad de materias, la extensión del contenido y todo el universo nuevo que la secundaria les ofrece.

A continuación, se expone el relato de una de las madres entrevistadas, que da cuenta de los cambios que debieron atravesar los estudiantes en relación a las singularidades de cada nivel de enseñanza:

Al pasar al secundario el nivel de exigencia fue otro, el cambio también, las demandas de las 10 u 11 materias que tenían al mismo tiempo, entonces no es lo mismo que 5 o 6 materias, es más light el primario. En el secundario todos exigen al mismo tiempo, los parciales eran uno atrás del otro, hay que organizarse en cuestiones de horarios, de tiempos (...) por ahí fue también esa la falla o la falta de un nivel a otro, en técnicas de estudio, en que se soltaran más o en tomarles más en forma oral, en ver cómo estudiaban ellos (...) creo que eso fue en primer año la crisis, la desesperación porque era todo nuevo, las exigencias, el ritmo de trabajo arduo, conocer a doce profesores, las formas de trabajo... (Entrevista familiar № 14).

\section{Falta de tiempo por parte de los familiares}

Ocho de los familiares entrevistados, como así también personal de la institución educativa, hicieron referencia a las múltiples ocupaciones de los adultos como uno de los principales motivos por los que se demandó apoyo pedagógico para los jóvenes. En este caso, la demanda se orienta más a cuestiones de índole socio-familiar. El contexto de aprendizaje resulta no solo un apoyo para los estudiantes, sino también para los padres y familiares, ya que las maestras asisten a los adolescentes durante sus tiempos libres y los familiares pueden dedicarse a sus ocupaciones laborales.

A continuación, se explicitan algunos fragmentos que sirven de ejemplo:

Porque yo como mamá, tenía muchas horas fuera de mi casa (...) por la situación económica actual, hace que los papás, ambos tengan que salir a trabajar y cantidad de horas fuera de la casa, como me pasa a mí (Entrevista familiar № 7).

Hoy en día los papás trabajan mucho y no tenemos ese suficiente tiempo para explicarles o enseñarles... se trabaja mucho afuera, muchos compromisos, muchas corridas en todo el día... entonces yo llego a casa sabiendo que él tiene los deberes hechos, si tiene una prueba ya sé que estudió (Entrevista familiar № 8).

Las expresiones de los familiares permiten dar cuenta de la relación que existe entre la demanda de apoyo pedagógico y la situación económica que atraviesan los adultos de la localidad. La búsqueda de nuevas fuentes de ingreso hace que los adultos tengan múltiples ocupaciones laborales y no tengan tiempo de ayudar a los jóvenes con las tareas escolares. 
Por su parte, las maestras de apoyo pedagógico y los agentes institucionales también expresaron en sus discursos que la situación económica actual y los cambios en las tipologías familiares se relacionan con la búsqueda por parte de los adultos de un profesional que apoye a sus hijos con las actividades académicas.

\section{Conocimientos insuficientes de los familiares}

Nueve de los familiares entrevistados mencionaron que el motivo por el que demandaron el contexto de apoyo pedagógico fue que consideraban que no tenían los conocimientos suficientes que, de acuerdo a su percepción, se requieren para poder ayudar a sus hijos con las tareas del colegio. Esto se debía a que no habían accedido al nivel de educación que estaba cursando el adolescente o a que no recordaban ciertos temas, contemplados dentro de los contenidos que se enseñan en el secundario, por haber cursado esos estudios muchos años atrás.

En algunos casos, al desconocer los contenidos impartidos en los cursos de educación secundaria, los familiares recurrieron a la ayuda externa brindada por las maestras de apoyo pedagógico. Esta solicitud refiere a aspectos de índole socio-familiar y da cuenta de la importancia que se asigna en el hogar a la educación de los jóvenes, en la que se depositan expectativas académicas, así como también expectativas sociales.

Tanto la directora de la institución como las maestras de apoyo pedagógico relacionaron el nivel educativo alcanzado por los familiares de los estudiantes con la demanda de clases particulares. También destacaron la importancia que los adultos le asignan a la educación, que se ve reflejada en la búsqueda de un profesional que pueda ayudar a los jóvenes con contenidos que escapan a sus conocimientos.

A continuación, se citan expresiones de los familiares para ilustrar esta subcategoría:

Hay muchas cosas que no entiendo. Lo que me explicaron a mí hace 25 años no es lo mismo de ahora... así que yo decidí, para no confundirlo más a él, decidí mandarlo a particular (Entrevista familiar № 2).

Yo no tengo un secundario y no sé explicarle a veces, porque hay cosas que no puedo, no las entiendo directamente, que me cuesta a mí (Entrevista familiar № 9).

En resumen, las demandas que tuvieron que ver con cuestiones de índole académica fueron principalmente aquellas en las que los familiares promovían la asistencia del adolescente a clases particulares para que fueran ayudados por un profesional a prepararse para las exigencias académicas de la institución educativa. La asistencia de los jóvenes a las clases de apoyo se vio atravesada también por otras circunstancias, tales como dificultades para realizar resúmenes, dependencia generada a las instancias 
de ayuda, dificultades para entender las explicaciones brindadas por los profesores y problemas de aprendizaje. Uno de los principales motivos por el que se solicitó el contexto de apoyo pedagógico fue por la necesidad de ayuda en el proceso de adaptación.

Cabe destacar que un grupo de familiares mencionó que los jóvenes asistían al apoyo para que se los ayudara a fortalecer su autoestima, confianza en sí mismos y a sentirse más seguros a la hora de enfrentarse a las situaciones de examen. En relación a estos motivos, de índole afectiva, si bien fueron mencionados en las entrevistas, los casos fueron escasos. Aunque se lo considera un aporte valioso, al no contar con datos suficientes para analizarlo de modo exhaustivo no se lo incluyó en el detalle de resultados.

Por último, se encuentran las cuestiones socio-familiares, que hacen referencia a la falta de tiempo y a los conocimientos insuficientes de los mayores para ayudar a los adolescentes con las tareas escolares.

En las categorías mencionadas - (a) ayuda al proceso de adaptación, (b) falta de tiempo por parte de los familiares y (c) conocimientos insuficientes de los familiares - se ha aludido a los motivos por los cuales los adultos demandaban la asistencia de sus hijos a clases particulares. A continuación, se buscan integrar percepciones más generales, que contemplan más bien una opinión sobre por qué el apoyo pedagógico es solicitado con recurrencia.

Ante la pregunta: ¿por qué considera que actualmente son cada vez más las familias que demandan que sus hijos asistan a clases particulares?, las percepciones generales se vinculan con aspectos situacionales. Las respuestas se orientaron hacia tres cuestiones principales: la masividad del grupo de clases, las exigencias institucionales y las dificultades para atender a la educación de los hijos.

En primer lugar, un gran número de familiares expresó una relación directa entre la cantidad de alumnos que presentan los cursos y la asistencia de los adolescentes a clases de apoyo. Para las madres entrevistadas, la masividad en el aula trae aparejadas dificultades para lograr la interacción entre los estudiantes y el profesor. Esto produce inseguridades, dudas y dificultades para aprender, para prestar atención y concentrarse. Los familiares recurren entonces a un profesor externo para que, mediante una atención más personalizada, los estudiantes puedan acceder a los conocimientos que no alcanzan durante el período de cursado. La mención al fenómeno de la masividad del grupo de clases deja entrever cuestiones personales y contextuales. Esta es una situación que preocupa particularmente a los padres de este curso, que se ven implicados en dicha situación. Los agentes institucionales también relacionaron la masividad de la clase con el incremento de la demanda de apoyo.

En segundo lugar, la percepción de los familiares sobre el incremento de la asistencia a clases particulares debido a las exigencias de la institución se relaciona con dos de los motivos por los que pidieron el apoyo para sus hijos. Por una parte, los estudiantes acudieron a las maestras particulares para contar con un apoyo profesional 
que los ayudara a cumplir con las exigencias propias del nivel secundario. Por otra parte, el hecho de que los familiares de los estudiantes desconocieran los temas que estudiaban sus hijos incidió en que demandaran esta ayuda particular.

En tercer lugar, si bien ninguno de los familiares entrevistados relacionó su demanda con un desligamiento de sus funciones como adulto en la educación de los adolescentes, muchos consideraron que el incremento de la asistencia a clases particulares se debe a que los padres depositan sus responsabilidades respecto a la educación de sus hijos en la figura de la maestra de apoyo. Las maestras entrevistadas también observaron que los familiares demandan sus servicios ante las dificultades que presentan para atender a sus responsabilidades en la educación de los jóvenes.

Este último aspecto podría verse representado en algunos de los motivos por los que los familiares expresaron que habían demandado el apoyo: la falta de tiempo y de conocimientos por parte de los adultos. El hecho de que trabajaran durante largas jornadas y no estuvieran mucho tiempo en sus hogares, sumado a la falta de conocimientos sobre ciertos contenidos curriculares, incidió en que los adultos —al no disponer del tiempo y de los recursos para ayudar a los jóvenes - confiaran a profesionales la asistencia y el seguimiento de la educación de sus hijos.

\section{REFLEXIONES FINALES}

El presente estudio permitió reflexionar acerca de las diversas demandas familiares a contextos de aprendizaje no formales en educación secundaria, al obtener información detallada en relación a algunos motivos por los que se solicitaron los contextos de apoyo pedagógico. El análisis de los datos obtenidos mostró que la asistencia de los jóvenes a los contextos de apoyo pedagógico se veía atravesada por cuestiones de índole académica, socio-familiar y afectiva; siendo las dos primeras las que aparecen con más fuerza en los relatos de los familiares.

Una de las principales demandas por cuestiones académicas surge por la necesidad de ayuda en el proceso de adaptación que implica el tránsito del nivel primario al secundario. El desfasaje entre un ciclo y otro, en relación a la extensión de los contenidos y a la apropiación de estrategias de estudio, se intenta remediar desde las familias con la búsqueda de apoyo pedagógico.

En relación a aquellas demandas por cuestiones de índole socio-familiar, se relatan las dificultades por parte de los padres para ayudar a los estudiantes en el aprendizaje de ciertos contenidos académicos - por no tener conocimientos sobre los temas o por no recordarlos - y la falta de tiempo para ayudar a los jóvenes. Esto se asemeja a uno de los planteos de Ventura et al. (2006), que consideran que uno de los principales motivos por los que se demanda este contexto se debe a que cumple la función de apoyo no solo a los estudiantes, sino también a las familias, al ofrecer un espacio para que los jóvenes hagan los trabajos que desde la institución educativa se solicita que realicen en sus hogares. Los planteos se corresponden también con los 
aportes de Runte-Geidel (2015), quien expresa que las clases de apoyo pedagógico cumplen una función más social que académica, ya que ofrecen servicios de ocupación en los tiempos libres de los jóvenes y apoyo para aquellas familias con múltiples ocupaciones laborales fuera de la esfera doméstica.

Un aspecto interesante refiere a las respuestas que dieron las madres ante la pregunta sobre los motivos generales por los que pensaban que se había incrementado la demanda de apoyo pedagógico. En algunas situaciones pudieron separar este interrogante de los motivos particulares por los que habían demandado el apoyo, y en otras no. Al referirse a la masividad de los cursos, hicieron mención principalmente a aspectos personales que motivaron sus demandas. Se observó que las personas expusieron sus motivos personales como cuestiones generales por las que se incrementa la asistencia de los jóvenes a clases particulares.

Desde la perspectiva socio-cultural se concibe al aprendizaje como un proceso social, co-construido en las interacciones y negociaciones con otros. Se entiende al aprendizaje como un proceso que se define en términos de interacciones sociales. Con lo anteriormente expresado, y de acuerdo a los resultados del estudio, no se puede dejar de considerar la idea de que los contextos de aprendizaje son diversos, creados por las personas y por sus expectativas (Rinaudo, 2014). La representación que cada persona internaliza en relación al contexto de apoyo pedagógico está atravesada por las necesidades y los motivos por los que demandó dicho espacio, tanto de carácter académico, afectivo o socio-familiar.

No es menor el hecho de que los espacios de apoyo pedagógico surjan de la necesidad de ayuda ante determinadas situaciones que los estudiantes no pueden resolver por sí solos. La propia denominación de dicho contexto de aprendizaje puede interpretarse como el sostén o andamiaje (Wood, Bruner y Ross, 1976) que necesitan los estudiantes para poder aprender los contenidos enseñados en la educación secundaria, a partir de la interacción y de la ayuda de otro.

Este estudio apunta a otras alternativas de aprendizaje y reconoce que este no solo acontece en instituciones educativas formales. Conocer más acerca de cómo se aprende en contextos de diferente naturaleza contribuye a repensar el rol del educador y permite contemplar posibles tareas y ámbitos de intervención en contextos no formales.

En la actualidad, la impronta de los enfoques socio-culturales y estudios derivados contribuyen a repensar en esta dirección, al dejar en claro que las personas no aprenden solas ni únicamente en ámbitos formales, sino que lo hacen en diversos contextos y siempre en interacción con otros. De esta manera, se abren nuevas posibilidades de desempeño de rol para los profesionales de la educación. El modo en que se desarrollan y construyen los aprendizajes en contextos no formales, como el investigado, produce novedosos y desafiantes campos de acción (Martín, Ciochetto y Vaja, 2016).

\section{REFERENCIAS}


Agalianos, A. (2011). The Challenge of Shadow Education. Private tutoring and its implications for policy makers in the European Union. Recuperado de http://www.nesse.fr/nesse/activities/reports/activities/reports/the-challenge-of-shadow-education-1

Bray, M. (1999). The Shadow Education System: Private Tutorig and Its Implications for Planners. París, Francia: UNESCO - International Institute for Educational Planning.

Bray, M., Adamson, B., y Mason, M. (2010). Educación comparada: enfoques y métodos. Buenos Aires, Argentina: Granica.

Buchmann, C. (2002). Getting ahead in Kenya: Social capital, shadow education, and achievement. En B. Fuller, y E. Hannum (Eds.), Schooling and Social Capital in Diverse Cultures (pp. 133-159). Ámsterdam, Holanda: JAI Press.

Manavella, A. M. (2016). La demanda familiar de contextos de aprendizaje extraescolares en Educación Secundaria (Tesis de Grado). Universidad Nacional de Villa María, Córdoba, Argentina.

Martín, R. (2014). Contextos de aprendizaje. Formales, no formales e informales. Ikastorratza, 12. Recuperado de http://www.ehu.eus/ikastorratza/12_alea/contextos.pdf

Martín, R., Ciochetto, E., y Vaja, A. (2016, noviembre). La diversidad de los contextos de aprendizaje en el desarrollo del rol del psicopedagogo. Trabajo presentado en la Jornada homenaje a Lev Vigostky, a 120 años de su nacimiento, Córdoba, Argentina.

Moraga, V. (2011). Somos mucho más que dos. Revista Rumbos, 28, 22-28.

Rinaudo, M. C. (2014). Estudios sobre los contextos de aprendizaje. Arenas y fronteras. En P. Paoloni, M. C. Rinaudo, y A. González Fernández (Comps.), Cuestiones en Psicología Educacional: cuestiones teóricas, metodológicas y estudios de campo (pp. 163-206). Tenerife, España: Sociedad Latina de Comunicación Social. Recuperado de http://issuu.com/revistalatinadecomunicacion/docs/cde01

Runte-Geidel, A. (2013). La incidencia de las clases particulares en España a través de los datos de PISA. Revista Española de Educación Comparada, 21, 249-282.

Runte-Geidel, A. (2015). La educación a la sombra en países del Sur de Europa y sus implicaciones sobre la Equidad. Revista Española de Educación Comparada, 25, 167-182.

Smitter, Y. (2006). Hacia una perspectiva sistémica de la educación no formal. Laurus, Revista de Educación, 12(22), 241-256.

Stake, J. (1998). Investigación con estudios de caso. Madrid, España: Morata.

Trilla, J., Gros, B., López, F., y Martín, M. J. (2003). La educación fuera de la escuela: ámbitos no formales y educación social. Barcelona, España: Ariel Edición.

Ventura, A., Neto-Mendes, A., y Costa, J. A. (2006). El fenómeno del apoyo al aprendizaje fuera de la escuela a través de un análisis comparado. Revista de currículum y formación del profesorado, 10(2), 1-19.

Wood, D., Bruner, J. S. y Ross, G. (1976). The role of tutoring in problem solving. Journal of Child Psychology and Psychiatry, 17, 89-100. 